\title{
Finding a job for dichloroacetate
}

\author{
Friedrich C. Luft
}

Published online: 21 December 2012

(C) Springer-Verlag Berlin Heidelberg 2012

Pyruvate dehydrogenase (PDH) is the first catalytic enzyme of the pyruvate dehydrogenase complex (PDC) located in the mitochondrial matrix of eukaryotes. The PDC provides the primary link between glycolysis and the citric acid cycle by catalyzing the irreversible conversion of pyruvate into acetyl-CoA by pyruvate decarboxylation. Acetyl-CoA may then enter the citric acid cycle to release nicotinamide adenine dinucleotide (NADH) which powers the electron transport process in the membrane to generate ATP. PDH performs another reaction within the PDC, namely a reductive acetylation of lipoic acid. Lipoic acid is covalently bound to dihydrolipoamide acetyltransferase, which is the second catalytic component enzyme of the PDC. PDH phosphorylation by pyruvate dehydrogenase kinase (PDK) inactivates the enzyme and subsequently the entire PDC. This state-of-affairs is reversed by PDH phosphatase (Fig. 1). The inhibition of the PDC by PDK will decrease the oxidation of pyruvate in the mitochondria and increase the conversion of pyruvate to lactate in the cytosol. There are four isoforms of PDK (PKD1, PKD2, PKD3, and PKD4). PDK4 is the predominant form of PDK in cardiac and skeletal muscle [1].

Pulmonary arterial hypertension is a lethal disease that is a secondary phenomenon in most cases, although primary genetic forms have been identified. The treatment options are poor. Recent studies indicate that not only is the pulmonary vascular resistance increased but also the hypertrophied right ventricle functions poorly [2]. Mechanistic studies

\section{F. C. Luft $(\square)$}

Experimental and Clinical Research Center, a joint cooperation between the Charité Medical Faculty and the Max-Delbrück

Center for Molecular Medicine, Lindenbergerweg 80,

13125 Berlin, Germany

e-mail: luft@mdc-berlin.de have necessarily relied on animal models. In an earlier report, Piao et al. [3] observed that PDK4 activity was increased in the right ventricle in two different rat models of pulmonary hypertension, monocrotaline administration, and pulmonary artery banding. In these models, glycolysis was increased, whereas oxygen consumption was decreased. The authors reported an improvement of right ventricular performance with chronic dichloroacetate (DCA) treatment.

In this issue, Piao et al. studied a genetic rat model of pulmonary hypertension, the Fawn-Hooded rat [4]. They conducted a microarray analysis of 168 genes related to glucose oxidation or fatty acid metabolism in the right ventricles of Fawn-Hooded rats and also in deceased patients with pulmonary hypertension. Genes encoding PDK4, aldolase B, Acyl-coenzyme A oxidase, and the Forkhead box protein $\mathrm{O} 1$ (FOXO1) were increased in the rats. FOXO1 is a transcription factor that plays important roles in regulation of gluconeogenesis and glycogenolysis by insulin signaling and is also central to the decision for preadipocytes to commit to adipogenesis. Moreover, FOXO1 regulates PKD4. The investigators also found increased PDK4 expression in human hearts from pulmonary arterial hypertension victims, while an inactive form of FOXO1 was decreased. Thus, the net effects in rats and humans with pulmonary hypertension were similar in kind. Also measured were other regulators of PKD4, such as HIF- $1 \alpha$ or FOXO3; however, these regulators were unchanged in the Fawn-Hooded rat model of pulmonary arterial hypertension. DCA applied acutely reduced proton production and increased glucose oxidation and ATP production. Given chronically, DCA decreased PDK4 and FOXO1, activated PDH, and increased glucose oxidation implying a corrected pathologic metabolic pathway. Cardiac output was significantly increased and treadmill 


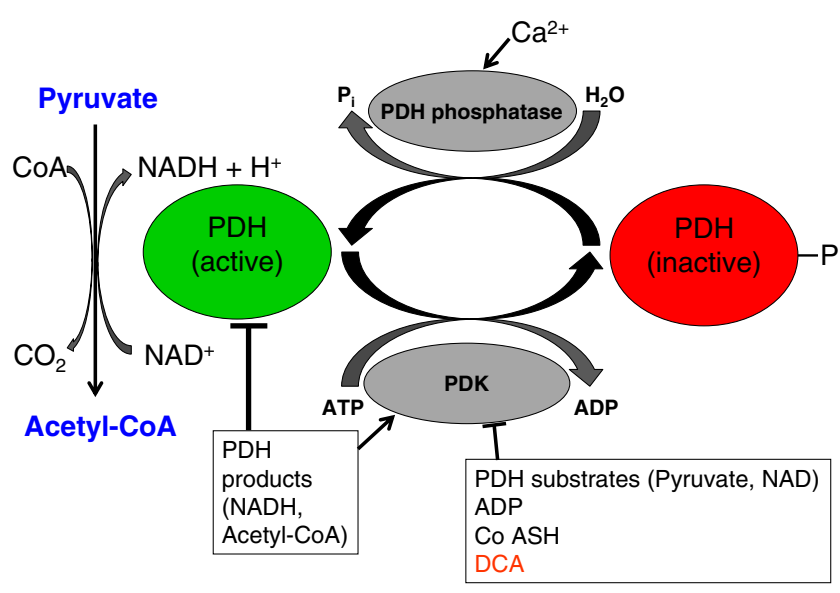

Fig. 1 Regulation of pyruvate dehydrogenase (PDH). The PDH activity is regulated by its state of phosphorylation, being most active in the dephosphorylated state. Phosphorylation of PDH is catalyzed by four specific PDH kinases, designated PDK1, PDK2, PDK3, and PDK4. The activity of these kinases is enhanced when cellular energy charge is high which is reflected by an increase in the level of ATP, NADH, and acetyl-CoA. Conversely, an increase in pyruvate strongly inhibits PDH kinases. Additional negative effectors of PDH kinases are ADP, $\mathrm{NAD}^{+}$, and $\mathrm{CoASH}$, the levels of which increase when energy levels fall. PDH phosphatase binds to the dehydrogenase complex, hydrolyzes the phosphorylated serine, reactivating $\mathrm{PDH}$. The phosphatase will remain associated with the dehydrogenase complex as long as the $\mathrm{NADH} / \mathrm{NAD}+$ and the acetyl-CoA/CoA ratios remain low. High concentrations of NADH or acetyl-CoA inactivate the phosphatase and cause it to dissociate from the pyruvate dehydrogenase complex. Insulin and $\mathrm{Ca}^{2+}$ ions activate the phosphatase

performance improved in Fawn-Hooded rats given the drug; however, right ventricular systolic pressure was not affected. The authors suggest a potential therapeutic role for PDK4 inhibitors in pulmonary arterial hypertension with right heart failure but do not advocate the use of DCA.

DCA is a salt of dichloroacetic acid $\left(\mathrm{CHCl}_{2} \mathrm{COOH}\right)$. The compound is well known and has a checkered clinical career. DCA has been studied as potential drug because the DCA ion stimulates pyruvate dehydrogenase activity by inhibiting PDK [5]. Thus, DCA decreases lactate production by shifting the metabolism of pyruvate from fermentation towards oxidation in the mitochondria. This property has led to trials of DCA for the treatment of lactic acidosis in humans [6-9]. A randomized controlled trial in children with congenital lactic acidosis found that while DCA was well tolerated, DCA was ineffective in improving clinical outcomes [7]. A separate trial of DCA in children with mitochondrial encephalomyopathy, lactic acidosis, and stroke-like episodes, a syndrome of inadequate mitochondrial function causing lactic acidosis, was halted early, as all 15 of the children receiving DCA experienced significant nerve toxicity without any evidence of benefit from the medication [8]. A randomized controlled trial of DCA in adults with lactic acidosis found that while DCA lowered blood lactate levels, it had no clinical benefit and did not improve hemodynamics or survival [9]. These results were a great disappointment to critical care clinicians.

DCA has also been considered as a cancer treatment. Cancer cells maintain a high rate of glycolysis but rely on anaerobic respiration that occurs in the cytosol leading to lactic acid fermentation rather than oxidative phosphorylation in the mitochondria for energy. This phenomenon is termed the Warburg effect, named after its discoverer. Malignant, rapidly growing tumor cells typically have glycolytic rates up to 200 times higher than those of their normal tissues of origin. The phenomenon occurs even if oxygen is plentiful. Christofk et al. identified an enzyme that gives rise to the Warburg effect [10]. The research team implicated the embryonic M2 isoform of pyruvate kinase (PKM2). Pyruvate kinase catalyzes the transfer of a phosphate group from phosphoenolpyruvate to ADP, yielding one molecule of pyruvate and one molecule of ATP. Tumor cells have been shown to express exclusively PKM2. PKM2 enables cancer cells to consume glucose at an accelerated rate. When tumor cells were forced to switch to pyruvate kinase's alternative form by inhibiting the production of PKM2, their growth was curbed. PKM2 is receiving much attention as a target. However, DCA has also been tested.

Bonnet et al. compared several cancer cell lines with normal cell lines and found that cancer cells had more hyperpolarized mitochondria and were relatively deficient in $\mathrm{Kv}$ channels. They reasoned that if this metabolic-electrical remodeling were an adaptive response, its reversal might increase apoptosis and inhibit cancer growth. They used DCA and found that DCA restored mitochondrial function, thus restoring apoptosis, allowing cancer cells to self-destruct and shrink the tumor [11]. Bonnet et al. concluded that DCA could be a promising selective anticancer agent by decreasing proliferation and inhibiting tumor growth without apparent toxicity. However, at sustained higher doses, generally $25 \mathrm{mg} / \mathrm{kg}$ /day orally or greater, there is increased risk of several reversible toxicities, especially peripheral neuropathy, neurotoxicity, and gait disturbance [12]. Rodent studies suggest that at high doses, DCA could even be carcinogenic [13]. Thus far, DCA has been disappointing as a therapeutic agent for patients. However, the metabolic targets first identified by Warburg and underscored by the present studies remain available for future drug development.

Respectfully, Friedrich C. Luft 


\section{References}

1. Bowker-Kinley MM, Davis WI, Wu P, Harris RA, Popov KM (1998) Evidence for existence of tissue-specific regulation of the mammalian pyruvate dehydrogenase complex. Biochem J 329(Pt 1):191-196

2. Rehman J, Archer SL (2010) A proposed mitochondrial-metabolic mechanism for initiation and maintenance of pulmonary arterial hypertension in Fawn-Hooded rats: the Warburg model of pulmonary arterial hypertension. Adv Exp Med Biol 661:171-185

3. Piao L, Fang YH, Cadete VJ, Wietholt C, Urboniene D, Toth PT, Marsboom G, Zhang HJ, Haber I, Rehman J et al (2010) The inhibition of pyruvate dehydrogenase kinase improves impaired cardiac function and electrical remodeling in two models of right ventricular hypertrophy: resuscitating the hibernating right ventricle. J Mol Med (Berl) 88(1):47-60

4. Piao L, Sidhu VK, Fang Y-H, Ryan JJ, Parikh KS, Hong Z, Toth PT, Morrow E, Kutty S, Lopaschuk GD, et al. (2012) FOXO1-mediated upregulation of pyruvate dehydrogenase kinase-4 (PDK4) decreases glucose oxidation and impairs right ventricular function in pulmonary hypertension. J Mol Med. doi:10.1007/s00109-012-0982-0

5. Stacpoole PW (1989) The pharmacology of dichloroacetate. Metabolism 38(11):1124-1144

6. Stacpoole P, Lorenz A, Thomas R, Harman E (1988) Dichloroacetate in the treatment of lactic acidosis. Ann Intern Med 108(1):58-63

7. Stacpoole P, Kerr D, Barnes C, Bunch S, Carney P, Fennell E, Felitsyn N, Gilmore R, Greer M, Henderson G et al (2006)
Controlled clinical trial of dichloroacetate for treatment of congenital lactic acidosis in children. Pediatrics 117:1519-1531

8. Kaufmann P, Engelstad K, Wei Y, Jhung S, Sano M, Shungu D, Millar W, Hong X, Gooch C, Mao X et al (2006) Dichloroacetate causes toxic neuropathy in MELAS: a randomized, controlled clinical trial. Neurology 66(3):324-330

9. Stacpoole P, Wright E, Baumgartner T, Bersin R, Buchalter S, Curry S, Duncan C, Harman E, Henderson G, Jenkinson S (1992) A controlled clinical trial of dichloroacetate for treatment of lactic acidosis in adults. The dichloroacetate-lactic acidosis study group. N Engl J Med 327(22):1564-1569

10. Christofk HR, Vander Heiden MG, Harris MH, Ramanathan A, Gerszten RE, Wei R, Fleming MD, Schreiber SL, Cantley LC (2008) The M2 splice isoform of pyruvate kinase is important for cancer metabolism and tumour growth. Nature 452(7184):230-233

11. Bonnet S, Archer SL, Allalunis-Turner J, Haromy A, Beaulieu C, Thompson R, Lee CT, Lopaschuk GD, Puttagunta L, Bonnet S et al (2007) A mitochondria-K + channel axis is suppressed in cancer and its normalization promotes apoptosis and inhibits cancer growth. Cancer Cell 11(1):37-51

12. Stacpoole PW (2011) The dichloroacetate dilemma: environmental hazard versus therapeutic goldmine - both or neither? Environ Health Perspect 119(2):155-158

13. DeAngelo AB, Daniel FB, Stober JA, Olson GR (1991) The carcinogenicity of dichloroacetic acid in the male $\mathrm{B} 6 \mathrm{C} 3 \mathrm{~F} 1$ mouse. Fundam Appl Toxicol 16:337-347 